\title{
Applying Image Fusion and Fuzzy Clustering Algorithms to Detect Changes in Synthetic Aperture Radar Images
}

\author{
Deepthi Maryala \\ M.Tech Student \\ Dept of ECE \\ Vardhaman College of \\ Engineering \\ Shamshabad, Hyderabad, India
}

\author{
J.Krishna Chaithanya \\ Associate Professor \\ Dept of ECE \\ Vardhaman College of \\ Engineering \\ Shamshabad, Hyderabad,India
}

\author{
T. Ramashri, Ph.D \\ Professor \\ Dept of ECE \\ Sri Venkateshwara University \\ Tirupathi, India
}

\begin{abstract}
Image change detection is a process in which two or more images of same scene that are captured at two different time instants are taken and changes are detected. Out of many categories of images, detection of changes in Synthetic Aperture Radar (SAR) images is a critical task because of the speckle noise present in these images. In this paper changes are detected in SAR images based on an image fusion strategy and fuzzy clustering algorithms. Image fusion is used to generate a difference image from the information obtained from mean ration and log ratio operator and Stationary Wavelet Transform (SWT) is used to decompose the sub bands and the two clustering algorithms namely fuzzy local information $\mathrm{C}$ means clustering (FLICM) algorithm and reformulated fuzzy local-information $\mathrm{C}$-means clustering algorithm (RFLICM) are considered which are applied on the fused image and the changed and unchanged areas are differentiated by forming clusters of similar and dissimilar elements. Experiments are carried out on the different images by applying FLICM and RFLICM algorithms.
\end{abstract}

\section{Keywords}

Synthetic aperture radar (SAR) images, Change Detection, clustering, Image Fusion, fuzzy local information C- means algorithm (FLICM), reformulated fuzzy local information Cmeans algorithm (RFLICM)

\section{INTRODUCTION}

Image change detection is a process that analyzes a pair of images which are acquired on the same geographical area at two different time instants. It has found important applications in remote sensing [1], flood monitoring, geographical map updating [2], medical diagnosis and video surveillance [3]. Remote sensing systems offers many advantages out of which one is the capability of repetitive coverage of a particular area at different times to study change detection process. Different types of sensors can be used to accomplish this task. Synthetic aperture radar (SAR) imagery [4] has found a major field of interest when compared to optical ones, due to its advantages such as operating in various weather conditions and are not affected by different sunlight conditions and cloud cover etc., but these SAR images contain some unwanted artifacts in the form of granules which is called as speckle noise. Due to the presence of this speckle noise, some of the crucial and important information of the images may be lost. In spite of its drawback change detection is clearly done due to its advantages. Many algorithms have been already proposed to detect the changes in SAR images. In this paper two aspects are related namely
1. Producing the difference images by fusing mean ratio operated image and log ratio operated image.

2. Operating the FLICM and RFLICM algorithms (which is insensitive to noise) to detect changed and unchanged areas.

\section{RELATED WORKS}

The main concept of FLICM and RFLICM was originally discussed by Maoguo Gong [1] where he proposed the unsupervised SAR change detection approach which used DWT based image fusion and fuzzy clustering algorithms. Francesca Bovola et al., [5] proposed a novel adaptive scale driven approach to detect changes in multitemporal SAR images which exploits information at various scales to improve accuracy and geometric fidelity of change detection map. Florentin Bujor et al,[2] proposed an approach that enables geophysicists to detect regions which contain spatial features or temporal changes. Florent Chatelin et al, [6] used a family of multivariate gamma distributions for multisensory SAR images which are referred to as MuMGDs. Amandine Robin et al, [3] provided an unsupervised method for sub pixel change detection by comparing $\mathrm{CR}$ time series with $\mathrm{HR}$ classification at a reference date; it does not require any a priori information but depends on reject of naïve mode. Lorenzo Bruzzone [7] proposed a novel automatic approach to detect changes in multitemporal remote sensing images using Bayesian theory, two theoretical approaches were used namely Reduced Parzen Estimate (RPF) technique which is used to get rough non parametric initial estimates of changed and unchanged pixels in difference image which are iteratively improved by Expectation Maximization (EM) algorithm and Markov Random Field (MRF) approach is used to generate the final change detection map. David Rey [8] concentrated on medical images where he proposed a new method to study multiple sclerosis lesion evolution between two images. Marcel Bose et al,[9] presented a technique to detect changes in MRI images. In this paper changes are detected in synthetic aperture radar (SAR) images using Stationary Wavelet Transform (SWT) based image fusion principle and fuzzy local information C- means algorithm (FLICM)[10],[11], reformulated fuzzy local information cmeans algorithm (RFLICM). SWT based image fusion technique and respective clustering algorithms are applied on SAR images and compare the outputs yielded for FLICM and RFLICM [1][10][11] algorithms to discriminate the changed areas from that of unchanged areas. 


\section{MOTIVATION}

Let us consider two images $\mathrm{X}_{1}$ and $\mathrm{X}_{2}$, where $\mathrm{X}_{1}$ denotes the original SAR image 1 and is given by $\mathrm{X}_{1}=\left\{\mathrm{X}_{1}(\mathrm{i}, \mathrm{j}), 1<\mathrm{i}<\mathrm{h}\right.$, $1<j<w\}$ and $X_{2}$ denotes original SAR image 2 and given by $\mathrm{X}_{2}=\left\{\mathrm{X}_{2}(\mathrm{i}, \mathrm{j}), 1<\mathrm{i}<\mathrm{h}, 1<\mathrm{j}<\mathrm{w}\right\}$ of size $\mathrm{hxw}$ which are acquired on two different dates with two time instants $t_{1}$ and $t_{2}$.

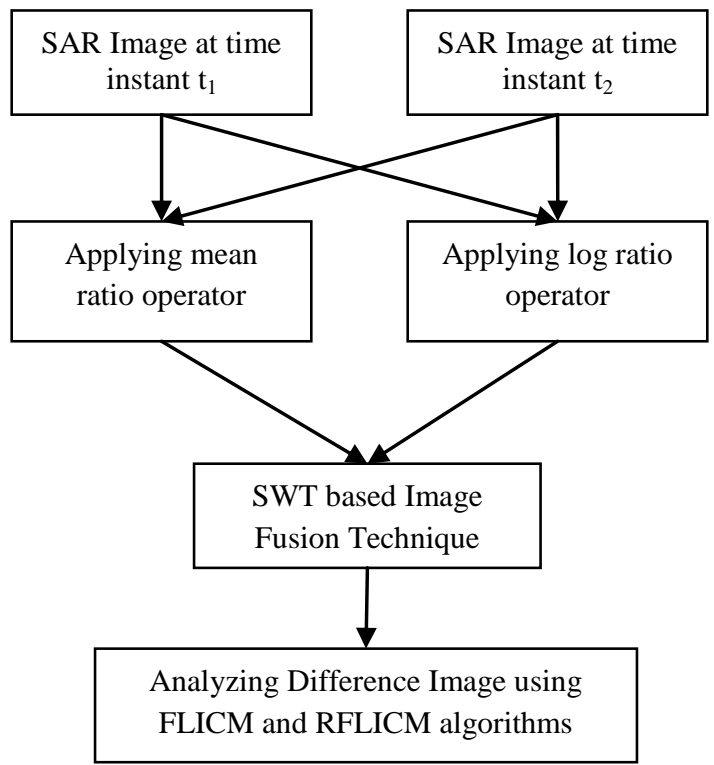

Figure 1. Flow Diagram of change detection approach

Figure 1 shows the actual flow diagram of the change detection approach. It can be categorized into two phases, the first phase involves obtaining information from mean ratio operator and $\log$ ratio operator and applying fusion rule on them to generate a difference image, and second phase includes analyzing the final fused image by using an FLICM and RFLICM algorithms.

\subsection{Generation of Difference Image using Image Fusion Technique}

Log normal model [1] was considered as a probability distribution function for SAR amplitude distributions and its intensity [4]. With the use of this log ratio operator [5][14] the speckle noise which is present in the SAR images will be transformed into additional noise component., and also log ratio operator enhances the pixels in weaker areas and vice versa due to which the changed area that is obtained may not be the actual changed region but mean while ratio mean detector is also used which will assume that even a small change in the scene will reflect as modified value of local mean value. However as for RMD (ratio mean detector) the unchanged area of the mean ratio image is quite rough, so in general it can be noticed that pixels which are changed will exhibit larger values and pixels that are unchanged exhibit low values, in other words it can be explained as restraining the unchanged area and enhancing the changed area.

The complementary information obtained by both mean ratio and $\log$ ratio images is taken by image fusion to generate a difference image. Mean ratio and log ratio operator formulae are given below.

$$
\begin{aligned}
& X_{m}=1-\min \left(\frac{\mu 1}{\mu 2}, \frac{\mu 2}{\mu 1}\right) \\
& X_{l}=\left|\log \frac{X_{2}}{X_{1}}\right|=\left|\log X_{2}-\log X_{1}\right|
\end{aligned}
$$

Here $\mu_{1}$ and $\mu_{2}$ denotes the local mean values of any image $X_{1}$ and $\mathrm{X}_{2}$, mean ratio image is denoted by $X_{m}$ and $\log$ ratio image is denoted by $X_{l}$. Mean ratio images and log ratio images are obtained by operating the original SAR images by using the above mentioned equations. Then image fusion technique is used employed, this technique generally takes complementary information from two or more images to get a most suitable fused image. To do the image fusion, a transform is required, transform such as discrete wavelet transform (DWT) [1] can be used which efficiently preserves the time and frequency components of an image but it has some drawbacks such as it suffers from poor directionality and due to the input signal shifts there may be unpredictable changes in the DWT coefficients in other words it can be said as shift sensitive. To overcome these disadvantages, the successor transform for DWT called as stationary wavelet transform (SWT) [12],[13] can be used, which has advantages such as redundant, shift invariant, linear and it gives better approximate values when compared to DWT. The image fusion technique can be described in two steps as follows, (1). Computing SWT of the source images, so that decomposition of the images can be obtained (2) Fusing the corresponding coefficients with the detail sub bands using fusion rule.

Low pass and high pass filters can be used to accomplish the task. Figure 2 shows how the image fusion rule is done using SWT, each source image that is $X_{m}$ and $X_{l}$ are decomposed into four sub bands namely $\mathrm{CA}, \mathrm{CH}, \mathrm{CV}, \mathrm{CD}$, where $\mathrm{CA}$ represents the approximate portion of the image, $\mathrm{CH}$ represents the horizontal portion of the image, $\mathrm{CV}$ represents vertical portion of the image and $\mathrm{CD}$ represents diagonal portion of the image. Profile features of the source image are denoted by approximate portion (CA) and important features such as edges and lines are denoted by $\mathrm{CH}, \mathrm{CV}$ and $\mathrm{CD}$ respectively. Approximate coefficients of $\mathrm{k}^{\text {th }}$ level can be obtained from low frequency sub bands and $(\mathrm{k}+1)^{\text {th }}$ level from high frequency sub bands. The main idea to use fusion rule is to restrain the unchanged area that is the back ground portion of the image and to enhance the changed area. Many fusion rules are available to use but they have some disadvantages such as unchanged area may become rough because of maximization of edge features, but here it is needed to enhance the foreground (changed) region without changing background information. In order to do this two fusion rules can be taken such as

(1). Rule of selecting average value of coefficients of low frequency band and

(2). Rule of selecting minimum local area energy coefficient for high frequency band respectively which are described using the formulae given below.

$$
\begin{gathered}
S_{C A}^{F}=\frac{S_{C A}^{m}+S_{C A}^{l}}{2} \\
S_{\varepsilon}^{F}(i, j)=\left\{\begin{array}{l}
S_{\varepsilon}^{m}(i, j), E_{\varepsilon}^{m}(\mathrm{i}, \mathrm{j})<E_{\varepsilon}^{l}(\mathrm{i}, \mathrm{j}) \\
S_{\varepsilon}^{l}(i, j), E_{\varepsilon}^{m}(\mathrm{i}, \mathrm{j}) \geq E_{\varepsilon}^{l}(\mathrm{i}, \mathrm{j})
\end{array}\right.
\end{gathered}
$$




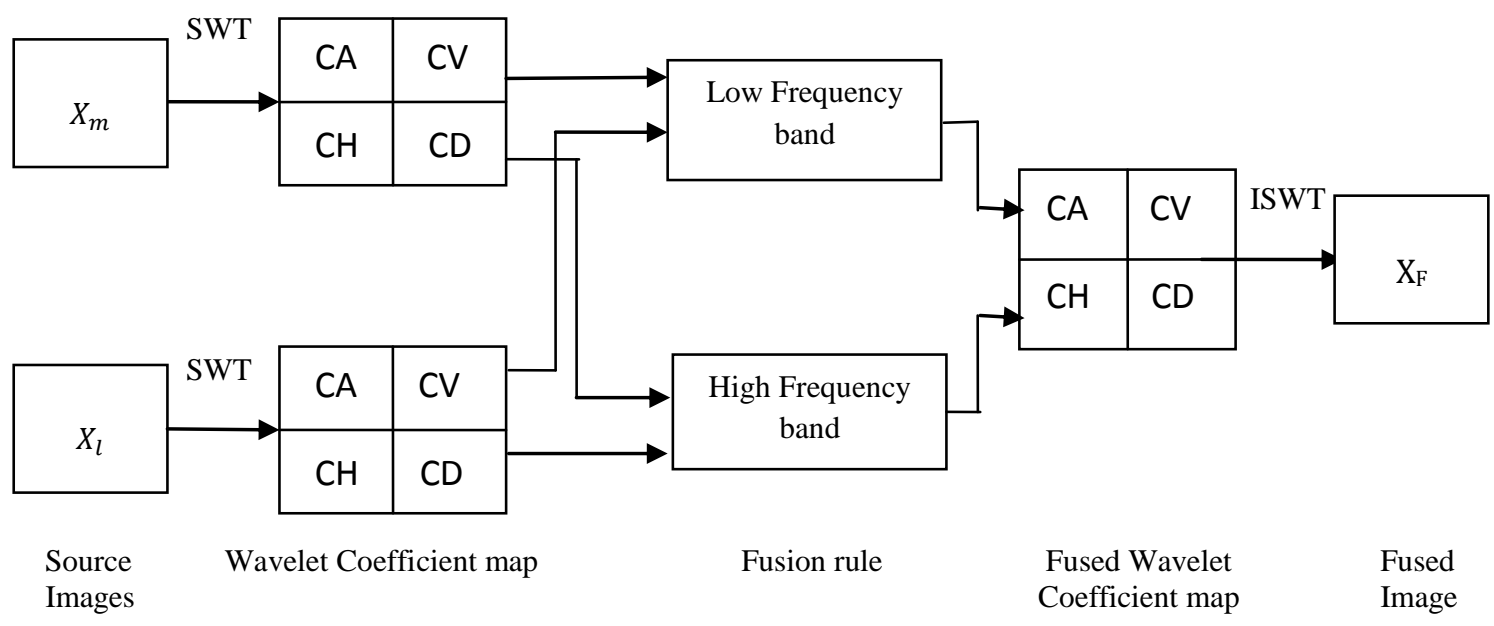

Figure 2. Block diagram for Image fusion technique based on Stationary Wavelet Transform

Mean ratio image and log ratio images are denoted by $m$ and $l$ respectively, $S_{C A}$ denotes the low frequency components, $S_{\varepsilon}(i, j)$ denotes other coefficients. As minimum local area energy coefficient is considered it is defined as

$$
E_{\varepsilon}(i, j)=\sum_{k \in N_{i, j}}\left[S_{\varepsilon}(k)\right]^{2}
$$

\subsection{Applying FLICM Algorithm}

Clustering is defined as classifying the objects in such a manner that samples of one cluster/class are similar to each other when compared to other class. In past decade, many clustering methods were used such as FCM [15][16] which can preserve the information of original image but it has a drawback that it is very sensitive to noise, to overcome this FCM_S was used but its computational complexity increased since it computes each time the neighborhood term. With the introduction of FGFCM (Fast Generalized FCM) [1] execution time was reduced but the main drawback was the introduction of a parameter to balance the effectiveness in preserving the details of the image and the sensitiveness to noise which is not that easy to implement. In order to overcome all the drawbacks in the traditional clustering algorithms, fuzzy local information c-means (FLICM) algorithm is introduced which uses a fuzzy factor to increase the performance of clustering, in this algorithm noise insensitiveness is guaranteed and image details can be easily retained with the usage of fuzzy local similarity measure. Fuzzy factor is given as follows:

$$
G_{k i}=\sum_{j \in N_{i}} \frac{1}{d_{i j}+1}\left(1-u_{k j}\right)^{m}\left\|x_{j}-v_{k}\right\|^{2}
$$

where spatial Euclidean distance between pixels $i$ and $j$ is represented by $d_{i j} . v_{k}$ is the prototype of center cluster $k, i^{t h}$ pixel is the center of local window and $j^{\text {th }}$ pixel is the neighboring pixel in adjacent window of $i^{\text {th }}$ pixel and fuzzy membership of the gray value $\mathrm{j}$ is given by $u_{k j}$, with respect to $k^{\text {th }}$ cluster. Here $G_{k i}$ is formulated without setting any artificial parameter. In addition to the above characteristics of FLICM [11] include noise immunity and being directly applied on original image. The objective function of FLICM can be defined using $G_{k i}$ as follows:

$$
J_{m}=\sum_{i=1}^{N} \sum_{k=1}^{c}\left[u_{k i}^{m}\left\|x_{i}-v_{k}\right\|^{2}+G_{k i}\right]
$$

where number of data items is denoted by $N$, number of clusters is denoted by $c$ and the Euclidean distance between object $x_{i}$ and the cluster center $v_{k}$ is given by $\left\|x_{i}-v_{k}\right\|^{2}$. Fuzzy membership partition matrix and cluster center calculations are given below

$$
\begin{aligned}
u_{k i} & =\frac{1}{\sum_{j=1}^{c}\left(\frac{\left\|x_{i}-v_{k}\right\|^{2}+G_{k i}}{\left\|x_{i}-v_{j}\right\|^{2}+G_{j i}}\right)^{1 /(m-1)}} \\
v_{k} & =\frac{\sum_{i=1}^{N} u_{k i}^{m} x_{i}}{\sum_{i=1}^{N} u_{k i}^{m}}
\end{aligned}
$$

Finally, the FLICM algorithm can be narrated as follows:

Step 1: Set the values for number of clusters $c$ fuzzy parameter $m$ and the stopping condition $\varepsilon$.

Step 2: Fuzzy partition matrix is initialized randomly.

Step 3: Loop counter b is set to zero.

Step 4: Compute the Cluster prototypes using the equation (9)

Step 5: Compute the fuzzy partition matrix using (8)

Step 6: If $\max \left\{U^{b}-U^{(b+1)}\right\}<\varepsilon$ then the process is stopped otherwise, increment $b$ that is $b=b+1$, and go to step 4 .

\subsection{Applying RFLICM Algorithm}

According to the analysis of the fuzzy factor $G_{k i}$, generally the gray level difference and spatial distance represents the local gray level information and spatial information in $G_{k i}$ these parameters change in accordance with the spatial distances from central pixel and for the neighborhood pixels having same gray-level value. Spatial distance and damping extent are inversely related, accurate estimation of the fuzzy factor is very much needed in order to suppress the noisy pixels. In order to overcome the drawbacks of spatial distance, local coefficient of variation is defined which is given below.

$$
C_{u}=\frac{\operatorname{var}(x)}{(\bar{x})^{2}}
$$

where intensity variance is denoted by $\operatorname{var}(x)$ and $\bar{x}$ is the mean in the local window of the image. Gray value homogeneity degree of local window which exhibits high 
values in noisy areas and low values in homogeneous areas is given by the value $C_{u}$. The results of the local coefficient of variation are very close in the homogeneous area or the area corrupted with noise. The local coefficient of variation between neighboring pixels and central pixel is relatively in accordance with the gray level difference between them which helps to exploit more local context information because local coefficient variation of each pixel is computed in the local window. The modified fuzzy factor $G_{k i}^{\prime}$ can be defined as follows:

$$
G_{k i}^{\prime}=\left\{\begin{array}{c}
\sum_{j \in N_{i}} \frac{1}{2+\min \left(\left(\frac{\mathrm{c}_{\mathrm{u}}^{\mathrm{j}}}{\mathrm{c}_{\mathrm{u}}}\right)^{2},\left(\frac{\mathrm{c}_{\mathrm{u}}}{\mathrm{c}_{\mathrm{u}}^{j}}\right)^{2}\right)} \\
\mathrm{X}\left(1-u_{k j}\right)^{m}\left\|x_{j}-v_{k}\right\|^{2}, \text { if } C_{u}^{j} \geq \bar{C}_{u} \\
\sum_{j \in N_{i}} \frac{1}{2-\min \left(\left(\frac{\mathrm{c}_{\mathrm{u}}^{\mathrm{j}}}{\mathrm{C}_{\mathrm{u}}}\right)^{2},\left(\frac{\mathrm{c}_{\mathrm{u}}}{\mathrm{c}_{\mathrm{u}}^{\mathrm{j}}}\right)^{2}\right)} \\
\mathrm{X}\left(1-u_{k j}\right)^{m}\left\|x_{j}-v_{k}\right\|^{2}, \text { if } C_{u}^{j}<\bar{C}_{u}
\end{array}\right.
$$

where the local coefficient of variation of the central pixel is given by $C_{u}, C_{\mathrm{u}}^{\mathrm{j}}$ is the local coefficient of variation of neighboring pixels and $\bar{C}_{u}$ is the mean value of $C_{\mathrm{u}}^{\mathrm{j}}$ which is located in the local window. $G_{k i}^{\prime}$ is the reformulated fuzzy factor which balances the local coefficient of variation and also gray level of the neighboring pixels. The algorithm is named as reformulated fuzzy local information $\mathrm{c}-$ means (RFLICM) algorithm because if there is any difference between the results obtained for local coefficient of variation by central pixel and neighboring pixel, the weightings added of the neighboring pixel in $G_{k i}^{\prime}$ will be increased in order to suppress the outlier, hence this RFLICM is more robust compared to its pre-existence that is FLICM. RFLICM and FLICM incorporate spatial and gray level information to find the tradeoff between noise removal and preserving the actual details. Finally with the help of new fuzzy factor $G_{k i}^{\prime}$ RFLICM algorithm can be summarized as same as FLICM algorithm as given above.

Step 1: Set the values for number of clusters $c$ fuzzy parameter $m$ and the stopping condition $\varepsilon$.

Step 2: Fuzzy partition matrix is initialized randomly.

Step 3: Loop counter b is set to zero.

Step 4: Compute the Cluster prototypes using the equation (9)

Step 5: Compute the fuzzy partition matrix using (8)

Step 6: If $\max \left\{U^{b}-U^{(b+1)}\right\}<\varepsilon$ then the process is stopped otherwise, increment $b$ that is $b=b+1$, and go to step 4 .

\section{FLOW CHART}

Figure 3 shows the pictorial representation of the clustering and detection process, as shown in the figure it starts with taking into consideration the fused image that is the image which is obtained after using SWT based image fusion technique. The blocks denoted in braces is the clustering process, where the values for number of clusters, fuzzy parameter and stopping condition are set and then cluster prototypes and fuzzy partition matrices are computed using necessary formulae. In the decision making box, the condition is kept which on satisfied stops the process or else goes to before step.

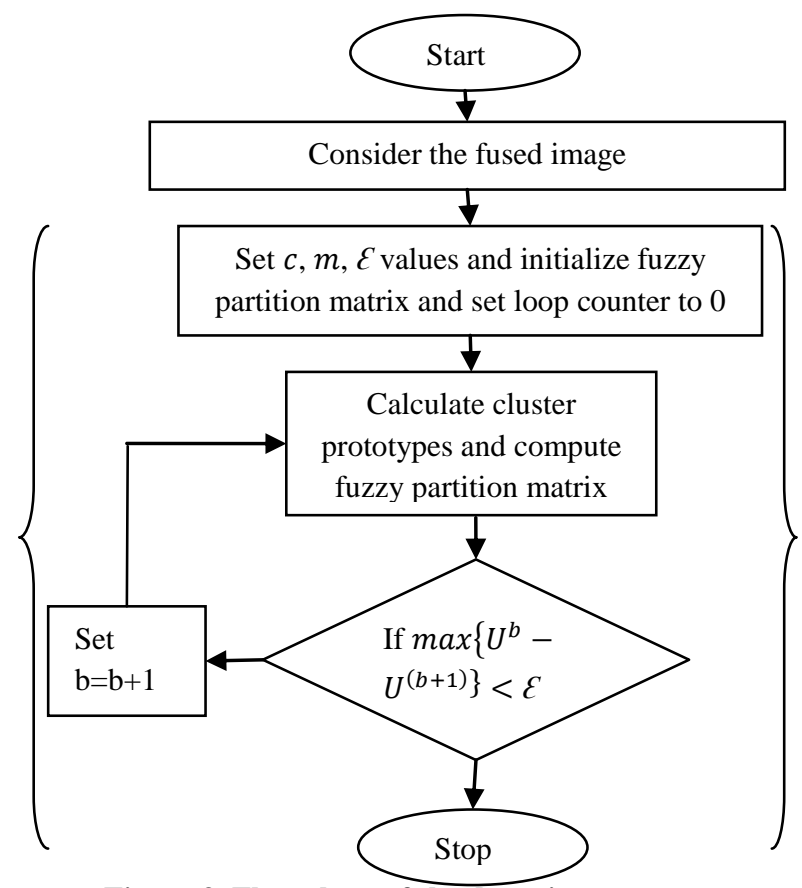

Figure 3. Flow chart of the detection process

\section{EXPERIMENTAL STUDY}

In order to validate the effectiveness of the proposed synthetic aperture radar based change detection approach, the performance of the proposed method can be studied using numerical results on a data set. The analysis of change detection is done as follows. First, calculate the false negatives (FN) nothing but changed pixels that are undetected. Second, calculate false positives (FP) that is unchanged pixels wrongly classified as changed. To calculate percentage correct classification (PCC), true positives (TP) and true negatives (TN) are also needed. TP is the number of pixels that are detected as the changed area in the reference image and result. TN gives the number of pixels that are detected as the unchanged area in both the reference image and the result. Here, kappa statistic is used to measure the accuracy or agreement. If the change detection map and the reference image are in complete agreement, then the kappa value is 1 . If there is no agreement among change detection map and reference image, then the kappa value will be 0 .

$$
P C C=\frac{(T P+T N)}{(T P+F P+T N+F N)}
$$

SAR images which are taken at different times are considered and mean ratio and log ratio operators are operated on those images. SWT based images fusion technique is applied in order to decompose the sub bands and ground truth image is generated. Here, Figures $4 \& 5$ are the original SAR images, figure 6 is the mean ratio operator image, figure 7 is the log ratio operator image, figure 8 shows the SWT image, figure 9 is ground truth image and figures 10 \& 11 are the corresponding FLICM and RFLICM images. Tabular format given below shows the change detection results of the considered image set. 


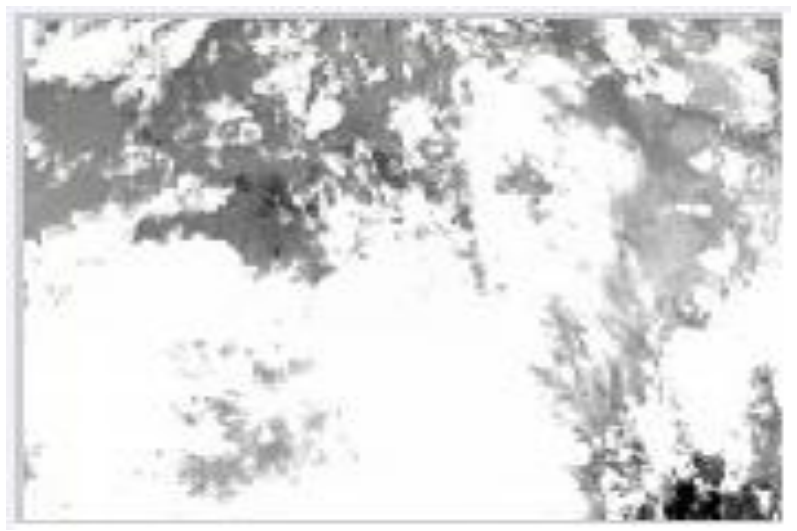

Figure 4. Original SAR Image 1

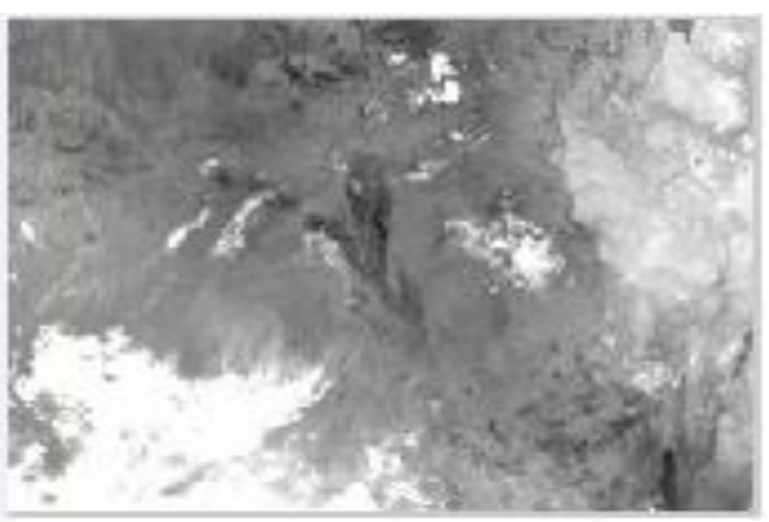

Figure 5. Original SAR Image 2

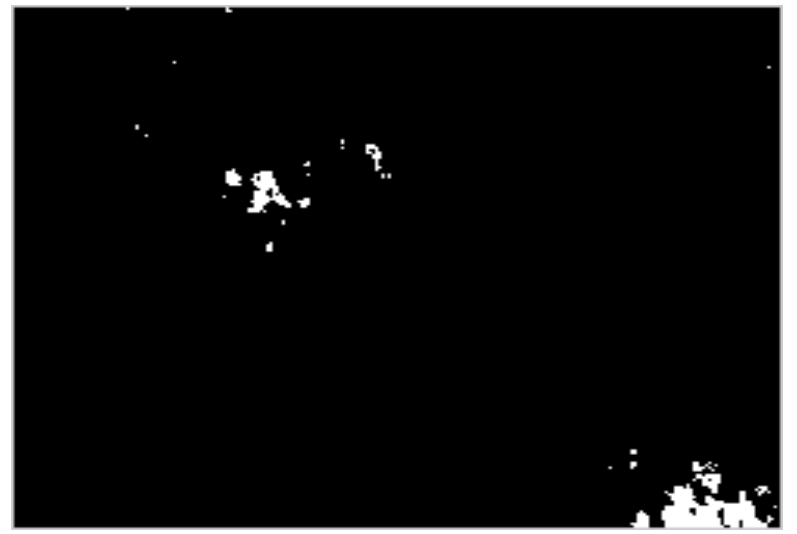

Figure 6. Mean ratio Operator Image

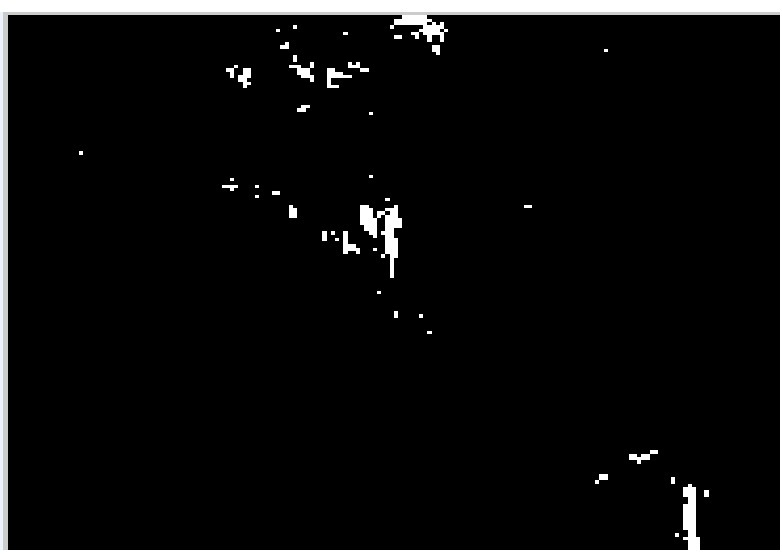

Figure 7. Log ratio Operator Image

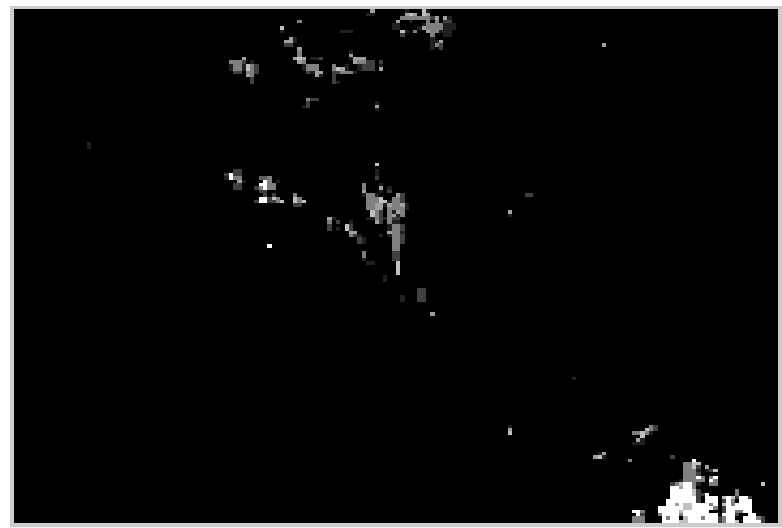

Figure 8. SWT Image

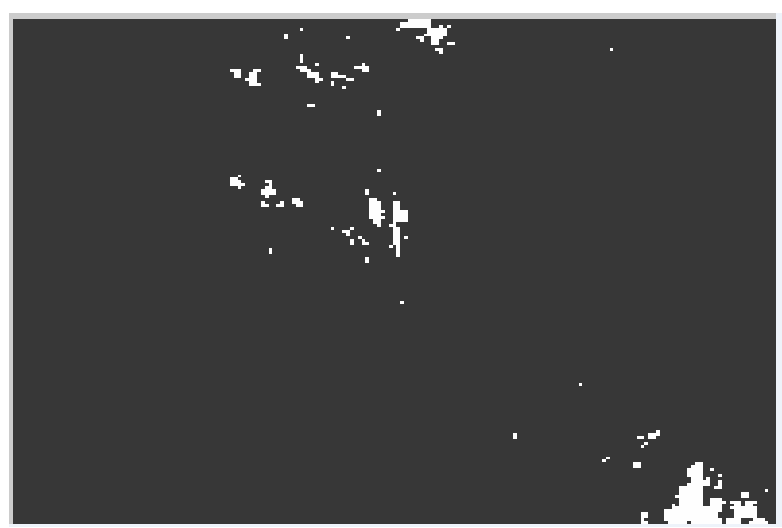

Figure 9. Ground Truth Image

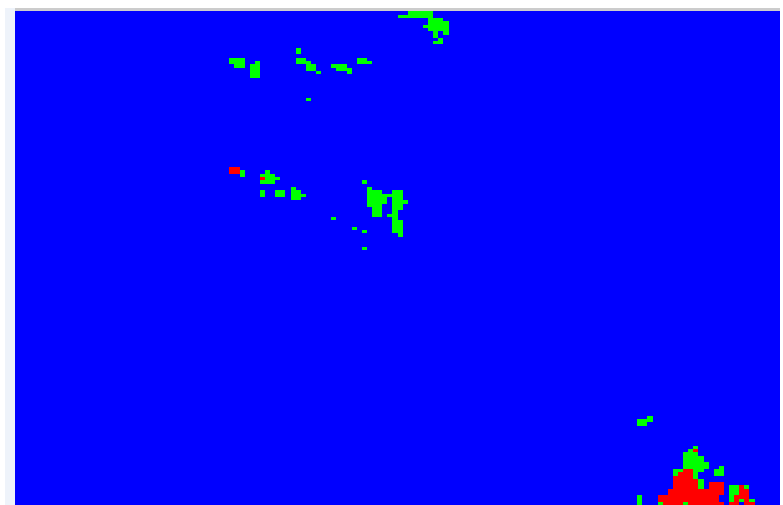

Figure 10. FLICM Image

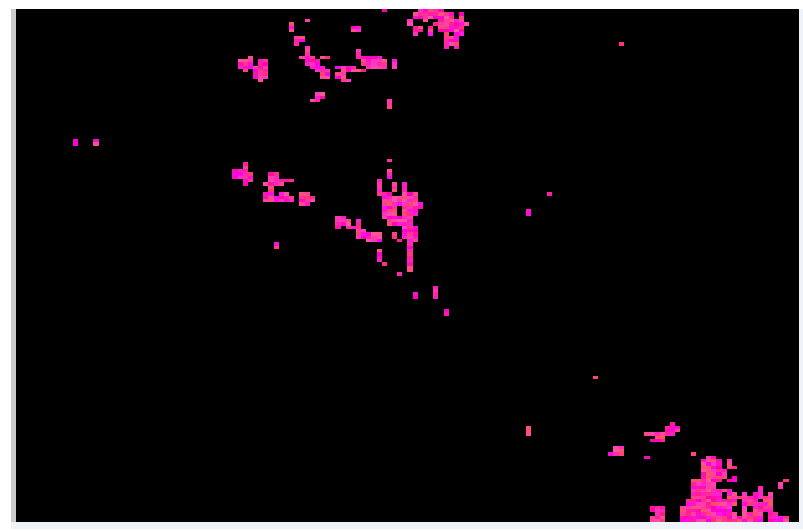

Figure 11. RFLICM Image 
Table 1 Change detection results of the considered image set

\begin{tabular}{|c|c|c|c|c|c|}
\hline $\begin{array}{c}\text { Operator or } \\
\text { Algorithm }\end{array}$ & TP & TN & FP & FN & PCC \\
\hline Mean- Ratio & 798 & 65337 & 14 & 603 & $78 \%$ \\
\hline Log- Ratio & 653 & 65191 & 160 & 748 & $73 \%$ \\
\hline FLICM & 621 & 65193 & 158 & 780 & $81 \%$ \\
\hline RFLICM & 858 & 65192 & 159 & 543 & $72 \%$ \\
\hline
\end{tabular}

\section{CONCLUSION}

In this paper, multi temporal SAR images are mainly concentrated for change detection without any disturbance caused by the speckle noise. Mean ratio and log ratio operators are considered from whose information fusion technique takes the complementary information to proceed further in generating a difference image, in this fusion technique SWT is used which overcomes the disadvantages of DWT such as shift sensitivity and poor directionality. After fusion technique is completed then clustering is done where FLICM and RFLICM algorithms are applied and change detection is observed. Results are evaluated and percentages are calculated. It is noticed that RFLICM yields better results than FLICM algorithm.

\section{REFERENCES}

[1] Maoguo Gong, Zihqiang Zhou, Jingjing Ma " Change detection in Synthetic aperture Radar Images based on Image Fusion and Fuzzy Clustering", IEEE Trans on image processing, Vol 21, No:4, April 2012

[2] F. Bujor, E. Trouvé, L. Valet, J. M. Nicolas, and J. P. Rudant, "Application of log-cumulants to the detection of spatiotemporal discontinuities in multitemporal SAR images," IEEE Trans. Geosci. Remote Sens., vol. 42, no. 10, pp. 2073-2084, Oct. 2004.

[3] A. Robin, L. Moisan, and S. Le Hegarat-Mascle, "An acontrario approach for subpixel change detection in satellite imagery," IEEE Trans. Pattern Anal. Mach. Intell., vol. 32, no. 11, pp. 1977-1993, Nov. 2010

[4] E. E. Kuruoglu and J. Zerubia, "Modeling SAR images with a generalization of the Rayleigh distribution," IEEE Trans. Image Process., vol. 13, no. 4, pp. 527-533, Apr. 2004.

[5] F. Bovolo and L. Bruzzone, "A detail-preserving scaledriven approach to change detection in multitemporal SAR images," IEEE Trans. Geosci. Remote Sens., vol. 43, no. 12, pp. 2963-2972, Dec. 2005.

[6] F. Chatelain, J.-Y. Tourneret, and J. Inglada, "Change detection in multisensor SAR images using bivariate Gamma distributions," IEEE Trans. Image Process., vol. 17, no. 3, pp. 249-258, Mar. 2008.

[7] L. Bruzzone and D. F. Prieto, "An adaptive semiparametric and context- based approach to unsupervised change detection in multi-temporal remotesensing images," IEEE Trans. Image Process., vol. 11, no. 4, pp. 452-466, Apr. 2002.

[8] D. Rey, G. Subsol, H. Delingette, and N. Ayache, "Automatic detection and segmentation of evolving processes in 3-D medical images: Application to multiple sclerosis," Med. Image Anal., vol. 6, no. 2, pp. 163-179, Jun. 2002.

[9] M. Bosc, F. Heitz, J. P. Armspach, I. Namer, D. Gounot, and L. Rumbach, "Automatic change detection in multimodal serial MRI: Application to multiple sclerosis lesion evolution," Neuroimage, vol. 20, no. 2, pp. $643-$ 656, Oct. 2003

[10] S. Krinidis and V. Chatzis, "A robust fuzzy local information C-means clustering algorithm," IEEE Trans. Image Process., vol. 19, no. 5, pp. 1328-1337, May 2010

[11] K.S.Tamilselvan and Dr.G.Murugesan "Automatic Tumour Detection in Brain Image using FLICM algorithm

[12] G.P Nason and B.W Silverman, "The Stationary wavelet transform and some statistical applications, Dept of Mathematics, UK.

[13] Bagawade Ramdas P,Bhagawat Keshav S, Patil Pradeep $\mathrm{M}$, “ Wavelet transform Techniques for Image Resolution Enhancement : A Study, ISSN 2250-2459, Volume 2, Issue 4, April 2012

[14] Y. Bazi, L. Bruzzone, and F. Melgani, "An unsupervised approach based on the generalized Gaussian model to automatic change detection in multitemporal SAR images," IEEE Trans. Geosci. Remote Sens., vol. 43, no. 4, pp. 874-887, Apr. 2005.

[15] M. Ahmed, S. Yamany, N. Mohamed, A. Farag, and T. Moriarty, "A modified fuzzy C-means algorithm for bias field estimation and segmentation of MRI data," IEEE Trans. Med. Imag., vol. 21, no. 3, pp. 193-199, Mar. 2002

[16] W. Cai, S. Chen, and D. Zhang, "Fast and robust fuzzy C-means clustering algorithms incorporating local information for image segmentation," Pattern Recognit., vol. 40, no. 3, pp. 825-838, Mar. 2007. 